\title{
Cancer prevention: a European approach
}

Within the European Community cancer causes more than 700000 deaths annually. There is a growing realisation, as the EC countries draw closer, that the prevention of cancer within the Community would benefit from a coordinated approach. The first step in cancer prevention is to determine causation and, although this has been clear for many years in the case of lung cancer, the causes of the other major cancers have still to be determined. Diet is thought to be a major contributor to the risk of cancer at many sites in the body; the range of dietary patterns within the EC is very wide, as is the range in incidence of cancer at the sites of interest. It would be sensible to conduct studies of the aetiology of these common diet related cancers at the continental level, rather than within the national boundaries as at present. A number of cancers are of great importance because of the very great impact of each individual case, rather than their overall incidence. An example is ovarian cancer in young women; this is the most common cause of cancer death in women under the age of 40 years and the impact of each case on the relatives, particularly the young children of such patients, is often devastating. These cancers are not common in numerical terms and they are not easily studied within individual countries: they would be more sensibly studied at the continental rather than the national level. It is likely that measures to prevent the diet related cancers will need to take account of the realities of the Common Agricultural Policy, under which huge subsidies are given to support the production of (and also to promote the consumption of) many items that are not necessarily beneficial to longterm health. For example, the CAP subsidy to tobacco producers within the EC exceeds the budget for the Europe Against Cancer campaign more than 100-fold. The EC also promotes the consumption of butter (over margarine) and other dairy and meat products.

Because of the obvious advantages of a European approach, the European group for research in Cancer Prevention (ECP) was set up in 1981 to promote European collaboration in the study of the causation and ultimately the prevention of cancer and to the mirror the work of EORTC in the field of cancer treatment. ECP is organised into working groups, namely

(a) diet and cancer;

(b) tobacco related cancers;

(c) sexual and hormonal factors and cancer;

(d) viruses, AIDS and cancer;

(e) breast cancer;

(f) colon cancer.

In addition there is a public education group which is studying the methods needed to put the message of cancer prevention across to the public. All of the working groups have projects involving multinational collaboration in progress and a number of these are approaching completion. In addition, the groups take turns to organise the annual ECP symposium devoted to themes of high priority for cancer prevention, including 'Tobacco and cancer' (1983), 'Hormones and sexual factors in 
human cancer etiology' (1984), 'Diet and human carcinogenesis' (1985), 'Concepts and theories in carcinogenesis' (1986), 'Preventive strategies for cancers related to immune deficiencies' (1987), 'International symposium on gastric carcinogenesis' (1988). A number of workshops have been organised; for example in 1987 ECP organised a workshop on the causation and prevention of colorectal carcinogenesis. The symposia and some of the workshops have been published in the ECP symposium series.

To coordinate this research effort ECP has a headquarters in Brussels a Scientific Committee and a Scientific Coordinator. For further details of ECP, please contact either the Scientific Coordinator (Dr A Giacosa, Istituto Scientifico per lo Studio ela Cura dei Tumori, Genova, Italy) or the Chairman of the Scientific Committee (Dr M J Hill, PHLS-CAMR, Porton Down, Salisbury, Wiltshire SP4 0JG), or the Brussels headquarters (Dr A Maskens, 62 Avenue Lambeau, 1200 Brussels, Belgium).

Public Health Lab Service Board,

MICHAEL HILL

Porton Down,

Salisbury,

Wilts SP4 OJG. 